\section{Omega-3 fatty acids and neuroinflammation in Alzheimer's disease: the unraveling of neurorestorative cell signaling}

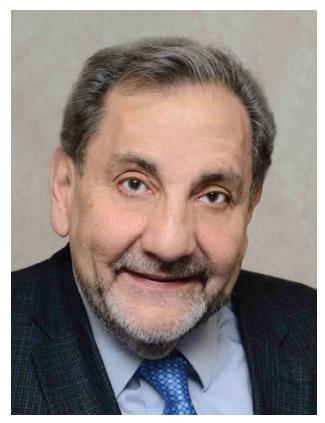

“...the evolving concept is not that neural cell survival fails due to biology gone awry, but rather to specific mechanisms that may be therapeutically harnessed."

Nicolas G Bazan*

First draft submitted: 11 March 2016; Accepted for publication: 18 March 2016; Published online: 3 May 2016

Alzheimer's disease (AD) is a challenge to civilization due to its increasing incidence and the lack of preventive approaches and effective therapies. The challenges include huge emotional burdens to families and caregivers, as well as the growing economic liability to the health care system. Given the present increased life expectancy, age has become the number one risk factor for $\mathrm{AD}$, and thus the incidence for this disease has grown because of it. The most prominent environmental risk factor for $\mathrm{AD}$ is traumatic brain injury and, in addition, several stroke and Parkinson's patients progressively transition to AD-type dementias. Currently there are no effective therapies for AD.

$\mathrm{AD}$ is a complex, multifactorial neurodegenerative disease characterized by progressive cognitive decline [1]. The early pathology includes enhanced neuroinflammation, which includes uncompensated oxidative stress and deregulated homeostasis with synaptic dysfunction and dendritic spine damage, followed by synaptic pruning and other events that lead to dementia. The two main pathological characteristics of $\mathrm{AD}$ are (A) formation of intracellular neurofibrillary tangles of the hyperphosphorylated tau protein [2], and (B) accumulation of $A \beta$ oligomers, leading to aggregates in amyloid plaques. These events are accompanied by microglia activation, astrogliosis and neurovascular unit impairments. Increased abundance of $A \beta$ oligomers in the intercellular space are among the earliest defects in AD that mediate a chain of neurotoxic events initiated by synaptic dysfunction, postsynaptic dendritic spine loss, and finally overt neurodegeneration.

Docosahexaenoic acid (DHA), a member of the omega-3 essential fatty acid family, is concentrated uniquely in the CNS as a component of all membranes, particularly synaptic, dendritic and photoreceptors [3]. In contrast, arachidonic acid (AA, an omega- 6 fatty acid) is distributed throughout mammalians tissues. DHA, which is esterified in phospholipids, follows a fate initiated by a phospholipase A2 (PLA2)-mediated cleavage. It then undergoes

*Neuroscience Center of Excellence, School of Medicine, Louisiana State University Health Sciences Center, New Orleans, LA 70112, USA; nbazan@lsuhsc.edu

\section{KEYWORDS}

- docosahexaenoic acid

- docosanoids

- neuroprotectin D1 
“...bioactive NPD1 shortage may not only be due to dietary restriction of the essential fatty acid precursor, but also to the liver as its supplier of building blocks of excitable membranes that control the replenishment of the membrane stores." enzymatic oxygenation via several pathways, generating bioactive docosanoids that promote homeostasis and neurorestoration. Docosanoids includes: neuroprotection D1 (NPD1; 10R,17Sdihydroxy-docosa-4Z,7Z,11E,13E,15Z,19Zhexaenoic acid), resolvins D1-D5 and maresins. 15-lipoxygenase-1 (15-LOX-1) is the enzyme that catalyzes the conversion of DHA into NPD1, a potent mediator of neuroprotection under conditions of uncompensated oxidative stress that, in turn, inhibits both proinflammatory and proapoptotic signaling enhancing prohomeostatic events (Figure 1).

Brain DHA content decreases during aging and particularly in $\mathrm{AD}$. In the CA1 hippocampal areas from donors with early-stage $\mathrm{AD}$ (no major neuronal loss yet), DHA is diminished and NPD1 is selectively decreased along with 15-LOX-1, the enzyme that catalyzes NPD1 synthesis [4]. These assessments were made in brain tissue that carefully controlled plaque and tangle count, age- and sex-matched controls as well as postmortem sampling interval (within 3-4 h) [5]. A drop in NPD1 was also found in the hippocampus of $3 \times \mathrm{Tg}-\mathrm{AD}$ mice between 4 and 12-13 months of age [5]. These observations were complemented by studies showing that the addition of NPD1 counteracted A $\beta$ toxicity in human brain neurons in culture. The implication of these findings is that the inability of the AD brain to efficiently synthetize NPD1 perturbs the neuroprotective effectiveness of this defensive mechanism, which includes the ability of NPD1 to downregulate amyloidogenic APP cleavage to shift the processing of the APP to the neuroprotective sAPP-alpha, with concomitant decrease in the formation of toxic $A \beta$ peptide [5]. These observations were made using human primary neuron/glia cultures overexpressing beta amyloid precursor protein ( $\beta$ APP) sw (Swedish double mutation APP695sw, K595N-M596L).

Thus NPD1 (and other docosanoids) are responsible for contributing at least in part to the bioactivity of DHA [6]. For example, NPD1 synthesis is upregulated as an early response to uncompensated oxidative stress, and to neurotrophins as specific adaptation-signaling responses, fostering resolution of inflammation and cell-survival signaling [3].

Because omega- 3 fatty acids sustain brain function, prevent cognitive decline and are potentially beneficial in $\mathrm{AD}$, the question to be asked is: why not supply them? This is followed by a more specific question: how can we supply them? The epidemiology of omega-3 fatty acids in $\mathrm{AD}$ has been inconclusive due, in some part to AD disease heterogeneity. Since brain DHA decreases during aging, one way to counteract this deficit may be by supplying either 18:3, eicosapentaenoic acid or DHA. The potential benefits of DHA supplementation or increased dietary intake of fish in slowing down cognitive decline has yielded unclear results. For example, some clinical trials suggest that DHA plays a role in slowing cognitive decline in elderly individuals without dementia; however, there is no clear evidence of prevention or treatment of dementias, including AD. In one study showing lack of effects on $\mathrm{AD}$, a subpopulation of patients with apoE4 polymorphisms did display benefits [7]. ApoE4 polymorphisms are increasingly recognized as a key factor in DHA significance in $\mathrm{AD}[8]$. On the other hand, animal studies have shown that DHA ameliorates AD disease pathology $[9,10]$. Also, a dietary omega-3 fatty acid restriction in an AD mouse model resulted in loss of synaptic markers, whereas DHA administration reversed this neuronal deficit. Moreover, other studies have shown that a high-fat diet with low DHA content increases tau and $A \beta$ levels, whereas a diet enriched in DHA reversed these changes, suggesting that shortage of DHA may be involved in the two main AD pathological features. Figure 1 depicts some of the beneficial bioactivity of NPD1: induction of a protective microglia phenotype as found in the retina [11]; induction of PPAR $\gamma$ and $s A P P \alpha$, as found in human brain cells [5]; transcriptional activation of nonredundant cRel/BIRC3 in the retina and brain [12]; activation of PP2A to down regulate oxidative stress-induce apoptosis at the mitochondrial level by dephosphorylation of $\mathrm{Bcl}-\mathrm{xL}$ that, in turn, sequesters BAX [13]; and restoration of Tau mislocalization (speculative). In addition, astrocytes and microglia clearly are involved in $\mathrm{AD}[5,14]$.

Nonetheless, the complex regulation of lipid metabolism, and how this affects disease pathology, is far from understood. Disturbances in DHA, NPD1 or other lipid mediators are linked to the initiation of the disease, and administration of NPD1 could have an impact in lowering $A \beta$ production and protection from neuronal insults. However, this might not be as straight forward as using dietary supplements of omega-3 fatty acids or DHA itself because between the ingestion and the uptake into the CNS are several events 


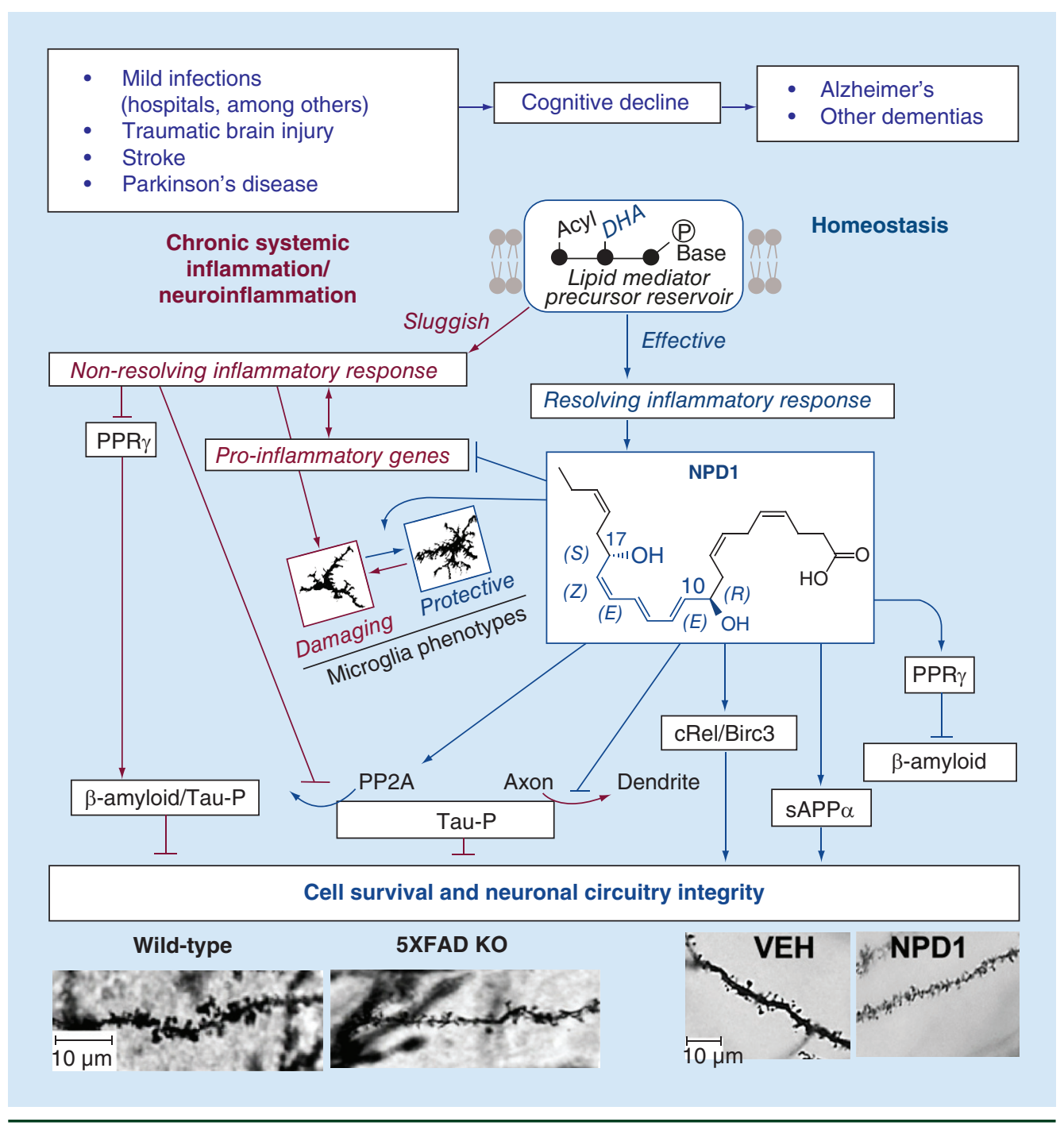

Figure 1. Counteracting pro- (blue) and anti- (red) homeostatic modulatory signaling in pathologies leading to cognitive decline and Alzheimer's disease. In the center is a membrane lipid mediator reservoir from where docosanoid precursors (e.g., DHA) are released either effectively or sluggishly. The docosanoid NPD1 is depicted, and pro- and anti-homeostatic targets are shown. One of the targets is microglia. One outcome is sustainment of the integrity of the synaptic circuitry. At the bottom are circuit impairments (depicts pruning of spines restored by NPD1) in an experimental model, the 5xFAD KO mouse (bottom right: VEH and NPD1 treatment in the 5xFAD KO mouse; protection of spines by the lipid mediator is shown).

KO: Knockout; NPD1: Neuroprotectin D1; VEH: Vehicle.

not well understood that might be critical and would explain, at least in part, the apparently contradictory outcome of several clinical studies based on supplementation of omega-3 fatty acids. The brain and retinal DHA uptake involves not-well-understood steps followed from gastrointestinal absorption. For example, how omega-3 fatty acids interact with the gut microbiome is not known, but there is crosstalk between gut microbes and the immune system that nurtures intestinal homeostasis. Impairments in the microbiome-host metabolic axis are involved in diseases, including neurodegenerations. Omega- 3 fatty acids $(18: 3,22: 6,22: 5)$ are transported to the liver by the portal system and then transported to the brain and retina [15]. Therefore bioactive NPD1 shortage may not only be due to dietary restriction of the essential fatty acid precursor, but also to the liver as its supplier of building

\section{"An additional \\ shortcoming has been the increasing recognition of the inadequacy of Alzheimer's disease animal models for pre-clinical testing of therapeutics because, for example, the rodent genome is devoid of the APOE genes that are risk factors for sporadic Alzheimer's disease."}


blocks of excitable membranes that control the replenishment of the membrane stores. The liver enzymes that supply DHA to the brain are dysfunctional in $\mathrm{AD}$, as reflected by the expression of peroxisomal d-bifunctional protein, which catalyzes the last step in the synthesis of DHA [16]. Moreover AD has multifactorial co-morbidities that include chronic systemic immune/inflammatory dysfunctions that heighten disease onset and progression $[17,18]$.

There are some exciting evolving concepts regarding the overall line of thought developed in the editorial: (1) the molecular logic of neuroprotection and neurorestoration involves neuron- and astrocyte-evoked release of potent docosanoid mediators from cellular membrane reservoirs in response to specific challenges: uncompensated oxidative stress, onset of homeostasis disruptions, and of conditions that set in motion neurodegenerations [6]; (2) there is a membrane encoding of information susceptible to regulation by neurotrophins in terms of concerted responses that release specific docosanoid mediators in response to defined challenges; (3) there is a "molecular logic" in the decision-making process of retrieving the precursors (omega-3 fatty acids) of docosanoids and related mediators, which engages a geneticallyprogrammed mechanism that defines the selective uptake/retention and storage specificity [19]; and (4) the failed adaptation response engages nonresolving inflammation set in motion by the perturbed balance of pro- and anti-homeostatic signaling quality and quantity of survival mediators (Figure 1). These fundamental issues of the biology of the CNS require a new scientific approach focused on neuronal cellularadaptation failure as the inability to evoke sufficient, specific survival-inducing docosanoid mediators from cell membranes. Of course, other mechanisms are critical, such as neurotrophin dysfunctions [20] and the incompletelyunderstood intracellular trafficking of proteins mediated by the retromer complex of proteins, among which mutations have been found in neurodegenerative diseases [21]. Therefore, the evolving concept is not that neural cell survival fails due to biology gone awry, but rather to specific mechanisms that may be therapeutically harnessed. Several apparently successful experimental drugs have failed advanced clinical trials, likely due to the complexity of the disease. We are currently realizing that clinical trial designs must include patient stratification as defined by genetic risks and disease phenotypes. An additional shortcoming has been the increasing recognition of the inadequacy of $\mathrm{AD}$ animal models for pre-clinical testing of therapeutics because, for example, the rodent genome is devoid of the APOE genes that are risk factors for sporadic AD.

In summary, omega-3 fatty acids, particularly DHA, clearly play a key role in neuroinflammation and Alzheimer's disease. The continued unraveling of the molecular logic of the cell signaling engaged will further our knowledge on novel, preventive and neurorestorative approaches for neurodegenerative diseases.

\section{Financial \& competing interests disclosure}

This work was supported by NIH grant P30 GM103340

(National Institute of General Medical Sciences to NG

Bazan). NG Bazan is the inventor of technologies/patents assigned to his university. The author has no other relevant affiliations or financial involvement with any organization or entity with a financial interest in or financial conflict with the subject matter or materials discussed in the manuscript apart from those disclosed.

No writing assistance was utilized in the production of this manuscript.

\section{References}

1 Ulrich JD, Holtzman DM. TREM2 Function in Alzheimer's disease and neurodegeneration. ACS Chem. Neurosci. 7(4), 420-427 (2016).

2 Mandelkow EM, Mandelkow E. Biochemistry and cell biology of tau protein in neurofibrillary degeneration. Cold Spring Harb. Perspect. Med. 2(7), a006247 (2012).

3 Bazan NG, Molina MF, Gordon WC. Docosahexaenoic acid signalolipidomics in nutrition: significance in aging, neuroinflammation, macular degeneration, Alzheimer's, and other neurodegenerative diseases. Annu. Rev. Nutr. 31, 321-351 (2011).
4 Lukiw WJ, Cui JG, Marcheselli VL et al. A role for docosahexaenoic acid-derived neuroprotectin D1 in neural cell survival and Alzheimer disease. J. Clin. Invest. 115(10), 2774-2783 (2005).

5 Zhao Y, Calon F, Julien C et al. Docosahexaenoic acid-derived neuroprotectin D1 induces neuronal survival via secretaseand PPAR $\gamma$-mediated mechanisms in Alzheimer's disease models. PLoS ONE 6(1), e15816 (2011).

6 Bazan NG. Is there a molecular logic that sustains neuronal functional integrity and survival? Lipid signaling is necessary for neuroprotective neuronal transcriptional programs. Mol. Neurobiol. 50(1), 1-5 (2014).

7 Quinn JF, Raman R, Thomas RG et al. Docosahexaenoic acid supplementation and cognitive decline in Alzheimer disease: a randomized trial. JAMA 304(17), 1903-1911 (2010).

8 Hennebelle M, Plourde M, ChouinardWatkins R, Castellano CA, Barberger-Gateau $\mathrm{P}$, Cunnane SC. Ageing and apoE change DHA homeostasis: relevance to age-related cognitive decline. Proc. Nutr. Soc. 73(1), 80-86 (2014). 
on F, Lim GP, Yang F et al.

Docosahexaenoic acid protects from dendritic pathology in an Alzheimer's disease mouse model. Neuron 43(5), 633-645 (2004).

10 Green KN, Martinez-Coria H, Khashwji H et al. Dietary docosahexaenoic acid and docosapentaenoic acid ameliorate amyloidbeta and tau pathology via a mechanism involving presenilin 1 levels. J. Neurosci. 27(16), 4385-4395 (2007).

11 Sheets KG, Jun B, Zhou Y et al. Microglial ramification and redistribution concomitant with the attenuation of choroidal neovascularization by neuroprotectin D1. Mol. Vis. 19, 1747-1759 (2013).

12 Calandria JM, Asatryan A, Balaszczuk V et al. NPD1-mediated stereoselective regulation of BIRC3 expression through cREL is decisive for neural cell survival. Cell Death Differ. 22(8), 1363-1377 (2015).

13 Antony R, Lukiw WJ, Bazan NG. Neuroprotectin D1 induces dephosphorylation of Bcl-xL in a PP2A-dependent manner during oxidative stress and promotes retinal pigment epithelial cell survival. J. Biol. Chem. 285(24), 18301-18308 (2010).

14 Rodríguez JJ, Butt AM, Gardenal E, Parpura V, Verkhratsky A. Complex and differential glial responses in Alzheimer's disease and ageing. Curr. Alzheimer Res. 13(4), 343-358 (2016).

15 Scott BL, Bazan NG. Membrane docosahexaenoate is supplied to the developing brain and retina by the liver. Proc. Natl Acad. Sci. USA 86(8), 2903-2907 (1989).

16 Astarita G, Jung K, Berchtold NC et al. Deficient liver biosynthesis of docosahexaenoic acid correlates with cognitive impairment in Alzheimer's disease. PLoS ONE 5(9), e12538 (2010).

17 Sarlus H, Eyjolfsdottir H, Eriksdotter M, Oprica M, Schultzberg M. Influence of allergy on immunoglobulins and amyloid- $\beta$ in the cerebrospinal fluid of patients with Alzheimer's disease. J. Alzheimers Dis. 48(2), 495-505 (2015).

18

Zhu M, Wang X, Hjorth E et al. Proresolving lipid mediators improve neuronal survival and increase $\mathrm{A} \beta 42$ phagocytosis. Mol. Neurobiol. 53(4), 2733-2749 (2015).

19 Rice DS, Calandria JM, Gordon WC et al. Adiponectin receptor 1 conserves docosahexaenoic acid and promotes photoreceptor cell survival. Nat. Commun. 6, 6228-6242 (2015).

20 Iulita MF, Cuello AC. Nerve growth factor metabolic dysfunction in Alzheimer's disease and Down syndrome. Trends Pharmacol. Sci. 35(7), 338-348 (2014).

21 Small SA, Petsko GA. Retromer in Alzheimer disease, Parkinson disease and other neurological disorders. Nat. Rev. Neurosci. 16(3), 126-132 (2015). 